\title{
Les Institutions De La Microfinance Entre La Responsabilité Sociale Et La Performance Financière: Cas Des Associations De Micro-Crédits
}

\author{
Laila Bennis, PhD \\ Ibn Tofail University
}

doi: 10.19044/esj.2016.v12n1p372 URL:http://dx.doi.org/10.19044/esj.2016.v12n1p372

\begin{abstract}
In recent years, microfinance becomes an engine of social and solidarity economy. The success of the microfinance sector and the media attention it generated as a tool to fight against poverty. It has increased the interest in the sector and attracted a number of private players (banks and investment funds) for which the financial profitability is crucial. This trend has enabled the sector to continue to grow and be more professional. Yet today this sector is subject to severe criticism on the risk of drifts of its institutions as over-indebtedness of clients and academic questions about the real impact of microcredit. The maturity of microfinance calls for growth and mastered firmly based on core principles (financial inclusion, customer protection, appropriate services etc.) in order to guard against the excesses of excessive commercialization which would be driven solely by the profit motive (financial profitability). Currently, microfinance institutions must demonstrate not only their reliability, cost efficient, their corporate and social responsibility. So complementarily between financial return and social performance is necessary for the sustainability of MFIs. Thus, a strong financial performance allows MFIs to have the capacity to pursue social objectives, and conversely, achieving social goals also improves profitability.
\end{abstract}

Keywords: Micro finance , microcredit -, social performance , financial profitability

Résumé

Au cours des dernières années, la micro finance devient une locomotive de l'économie sociale et solidaire. Le succès du secteur de la micro finance et l'engouement médiatique qu'il a suscité en tant qu'outil de lutte contre la pauvreté, ont renforcé l'intérêt pour ce secteur et ont attiré un certain nombre d'acteurs privés (banques et fonds d'investissement) pour 
lesquels la rentabilité financière est cruciale. Cette tendance a permis au secteur de poursuivre sa croissance et se professionnaliser davantage. Pourtant, aujourd'hui ce secteur fait objet des critiques sévères sur le risque de dérives de ses institutions comme le surendettement des clients et des questionnements académiques sur l'impact réel du microcrédit. La maturité de la micro finance appelle à une croissance maitrisée et reposant solidement sur ses principes de base (inclusion financière, protections des clients, services adaptés etc.) afin de se prémunir contre les dérives d'une commercialisation à outrance qui serait mue uniquement par la recherche du profit (rentabilité financière). Actuellement, les institutions de micro finance (IMF) doivent faire preuve, non seulement de leur fiabilité, rentables financièrement, de leur responsabilité sociale et sociétale (objectif majeur de l'économie sociale et solidaire) (Performance Sociale). Et donc une complémentarité entre la rentabilité financière et la performance sociale est nécessaire pour la pérennité des IMF. Ainsi, une performance financière solide permet aux IMF d'avoir les capacités de poursuivre leurs objectifs sociaux, et inversement, la réalisation de leurs objectifs sociaux permet aussi d'améliorer la rentabilité financière.

Mots clés: Micro finance, microcrédit, performance sociale, rentabilité financière

\section{Introduction}

Le terme de micro-finance est utilisé pour désigner les services financiers offerts aux individus de tout genre qui, traditionnellement, ont des difficultés d'usage ou d'accès aux services financiers ou sont complètement exclus du système financier formel. Le manque d'accès au financement institutionnel affaiblit nécessairement la sécurité financière de ces personnes à travers le monde.

La microfinance a vu le jour pour permettre l'inclusion des personnes exclus du système bancaire classique en leur offrant des services financiers (prêt, épargne, ...). Elle est considérée comme un moyen de lutte contre la pauvreté dans les pays en développement, à travers le financement des activités génératrices de revenus pour les ménages pauvres.

Pour répondre au souci d'inclusion, les institutions de la microfinance (IMF) se sont basées sur :

Un système de solidarité et la participation par exemple dans les systèmes coopératifs, chacun est membre et participe à la gestion de l'institution et considère que l'institution de microfinance lui appartient.

- $\quad$ Des services pour les exclus : les services ont été pensés et adaptés pour les besoins d'une population marginalisée économiquement ou socialement. 
- $\quad$ La proximité ${ }^{132}$ avec les bénéficiaires : une proximité géographique, proximité sociale dans la recherche d'une réduction des barrières entre les clients et une proximité temporelle se traduisant par des contacts fréquents entre l'institution et ses clients par des remboursements réguliers ou de fréquentes séances de formation et d’échanges.

Grâce à ces principes, la microfinance a connu un succès réel et beaucoup de projets et des initiatives, expérimentés dans de nombreux pays, ont gagné en puissance. La phase de croissance et la multiplication des expérimentations ont apporté la preuve qu'on pouvait servir les "nonbancables". Les IMF ont alors le plus souvent affiché leur mission de lutter contre la pauvreté, d'insérer les exclus dans les rouages de l'activité économique et de leur rendre leur dignité.

La microfinance permet de relancer l'entrepreneuriat local. Pour réaliser cette mission de façon durable et positive pour la population, la microfinance doit marier à l'objectif social, un objectif d'équilibre économique.

Les services financiers offerts par la microfinance concernent :

- $\quad$ Les services d'épargne qui permettent aux personnes de constituer un stock sécuritaire de liquidités, permanent, saisonnier ou temporaire pour une utilisation ultérieure et d'obtenir en plus, dans certains cas, des intérêts sur leurs dépôts.

- $\quad$ Les services de crédit, connus par le concept "micro-crédit", qui permettent l'utilisation d'un revenu anticipé pour l'investissement ou la consommation actuelle.

- Les produits de micro-assurances pour les personnes n'ayant pas de couverture sociale et d'autres formes d'assurances.

- D’autres services annexes se développent également qui concernent le crédit-bail, les transferts de fonds, ...

L'activité principale des institutions de microfinance est le microcrédit. Il consiste en l'attribution de prêts de faible montant à des entrepreneurs ou des artisans qui ne peuvent accéder aux prêts bancaires classiques. Le micro-crédit se développe surtout dans les pays en développement, où il permet de concrétiser des microprojets favorisant ainsi l'activité et la création de richesse mais se pratique aussi bien dans les pays développés ou en transition.

Au Maroc, le secteur du micro crédit s’est développé rapidement, Les premiers programmes de petits prêts destinés à financer les activités économiques des personnes à bas revenus ont démarré dans les années 19931994. Ce n’était qu’en 1996 que l'expérience internationale en matière de

\footnotetext{
132 - La proximité accroît la confiance, réduit les asymétries d'information et atténue les
} barrières sociales entre les clients et l'institution. 
microfinance a commencé à être connue dans le pays. A partir de cette date on a assisté à des créations d'institutions de microfinance, à l'application de nouveaux programmes, à l'innovation en matière de produits et services, à l'application de nouvelles méthodes...

Pourtant, à l'heure actuelle, les objectifs sociaux des institutions de microfinance (IMF) sont souvent questionnés mis en doute au niveau international et on s’interroge sur la population réellement touchée, sur la combinaison à la fois des objectifs sociaux et des objectifs de pérennité des IMF. Seules les études d'impact des produits de microfinance sur la population peuvent répondre à cette interrogation.

Dans notre article nous allons essayer de répondre à la question de savoir si les IMF peuvent être rentable financièrement sans pour autant délaisser leur raison d’être qui est la lutte contre la pauvreté et l'exclusion sociale.

\section{La micro-finance, un pilier de l'économie sociale et solidaire}

L'économie sociale et solidaire associe au développement économique une finalité sociale et sociétale. Elle se décline dans de très nombreux domaines qui ont comme dénominateur commun l'innovation sociale, des logiques participatives de fonctionnement et un fort ancrage territorial.

La micro-finance fait partie des domaines de l'économie sociale et solidaire. Elle a fait la preuve de deux idées principales. La première c'est qu'on peut concevoir des services financiers de proximité adaptés à des populations exclues du système financier formel, et qui ont un véritable impact sur leur développement économique et social, et la seconde c'est que les organisations qui offrent ces services peuvent être viables.

La microfinance est la forme de finance qui s’adresse en priorité aux exclus du système bancaire classique et de l'économie dominante. Aujourd'hui, après deux décennies d'une microfinance pionnière et triomphante, le secteur se trouve confronté pour la première fois à une crise en profondeur, fruit d'une marche forcée vers la rentabilité. De ce fait il faut chercher à promouvoir une forme particulière de micro-finance : la finance solidaire.

Il s’agit de refonder la microfinance sur la notion de solidarité : tisser des liens sociaux entre les clients eux-mêmes et entre les clients et l'institution, créer et consolider le capital social au sein de la clientèle. A travers la question de l'épargne et du crédit, c'est donc une autre approche du développement local qui se fait jour.

L'internationalisation de l'économie a fait basculer l'économie mondiale d'un modèle Etat/ Nation à un modèle Etat/Région. Les systèmes financiers internationaux se sont alors tournés vers les entreprises 
multinationales, acteurs de la région. Les systèmes bancaires locaux ont industrialisé leur production en devenant des entreprises bancaires comme les autres. Les finances solidaires ont alors pris en charge de plus en plus le financement des très petites entreprises et des entreprises émergentes de l'économie solidaire. Elles répondent désormais à des besoins financiers que le système financier a récusé et pour lesquels il ne dispose ni du mode d’organisation ni du savoir-faire adapté.

En réalité, avec la montée des inégalités, y compris au sein du salariat, depuis les années 90, on a assisté à un renouvellement de la finance solidaire $^{133}$.

Mais la micro-finance démontre aussi que le marché n'apporte pas toutes les solutions. En effet, le lancement d'institutions de micro-finance ne peut presque jamais se faire sans des donations publiques et sans la bienveillance des autorités. La micro-finance, à son échelle, est une réponse pragmatique à l'opposition apparente entre solidarité collective et sens de l'initiative individuelle.

\section{Le micro-crédit : genèse et développement}

Le micro-crédit consiste à fournir, à une population exclue du système bancaire classique, des prêts à court terme pour le financement des activités génératrices de revenus. Il a été initié en 1975 par le professeur de l'économie à l'université de Chittagong au Bangladesh Mohamed YUNUS $^{134}$. C'est dans les années 80 que le micro-crédit s'est développé grâce aux efforts de deux pays pionniers dans ce domaine, le Bangladesh avec la GRAMEEN BANK et la Bolivie avec la Banco Sol.

Les micro-crédits et même s'ils différent d'une institution de microcrédit à une autre et d'un pays à un autre, disposent de caractéristiques communes :

- Un faible montant du crédit ${ }^{135}$ : Le montant accordé aux clients est faible du fait que les besoins monétaires de la population ciblée par le micro-crédit

\footnotetext{
133 - La Grameen Bank octroie des prêts sans épargne préalable, ce qui a fait apparaitre tout le mouvement du microcrédit.

- L’épargne solidaire citoyenne apparaît dans le milieu des années 1980, en Europe et au Québec pour lutter contre le chômage et l'exclusion.

- L'investissement socialement responsable en lien avec l'aspect environnemental du développement durable complète la panoplie des interventions financières que, dans le langage commun, on peut qualifier de finance solidaire, de finance éthique.

134 - Selon Mohamed YUNUS, “Le micro-crédit c'est aider chaque personne à atteindre son meilleur potentiel. Il n’évoque pas le capital monétaire mais le capital humain. Le microcrédit constitue avant tout un outil qui libère les rêves des hommes et aide même le plus pauvre d'entre les pauvres à parvenir à la dignité, au respect et à donner un sens à sa vie."

135 - Au Maroc la loi sur le micro-crédit a fixé le plafond de micro-crédit à 50.000 MAD.
} 
sont modestes au vu de la situation précaire des pauvres qui ne leur permet pas de penser à des projets à forte intensité capitalistique ${ }^{136}$.

- La courte durée de remboursement : Les micro-crédits sont des crédits du court terme. En effet, la réussite des projets financés est due aux échéances proches et toutes difficultés de remboursement sont détectées au moment opportun, ce qui facilite le recouvrement de ces échéances.

- Une clientèle spécifique : Le micro-crédit a été créé pour servir les populations pauvres qui sont exclus du système bancaire classique. Il cible tout client pauvre et actif, qui veut créer une activité génératrice de revenu, qui ne dispose pas de capital nécessaire, qui ne présente aucune garantie réelle, et qui aux yeux des banques commerciales insolvable. Parmi ces clients pauvres, on trouve un pourcentage très important des femmes ${ }^{137}$.

- Un coût onéreux : Le taux d'intérêt est au centre des débats qui se déroulent autours de la microfinance ${ }^{138}$. Le micro-crédit est donc réputé être très onéreux comparativement aux crédits classiques offerts par le système financier traditionnel.

- Une destination à une activité génératrice de revenu : La destination principale du micro-crédit est la création ou l'extension d'une activité génératrice de revenu; il s'agit donc d'un crédit professionnel, favorisant l'insertion économique des personnes démunies dans la vie professionnelle, bien que ces dernières années, on parle de micro-crédit à la consommation, et de micro-crédit pour l'amélioration du logement.

- Des techniques d'octroi hors normes : Face à l'absence des garanties, les IMF mettent en place un système basé sur des techniques substituables à la sûreté réelle comme des relations de proximité avec les clients, l’octroi des crédits à des groupes solidaires et le recours à la technique du crédit progressif.

Au Maroc, le secteur du micro-crédit est relativement jeune. Les premiers programmes de petits prêts destinés à financer les activités économiques des

\footnotetext{
136 - Cette restriction a permis de segmenter le marché du crédit : les associations de microcrédit servent donc essentiellement les petites activités génératrices de revenu et les microentrepreneurs.

137 - Le micro-crédit touche des secteurs faiblement capitalisés employant souvent une main d'œuvre féminine ; d'ailleurs l'histoire de Mohammad Yunus et de la Grammen Bank a commencé avec des femmes qui représentent $97 \%{ }^{137}$ des emprunteurs de la banque, et les programmes de micro-crédit continuent à privilégier la femme.

138 - C'est le principal point de divergence entre deux visions théoriques; la vision institutionnelle qui propose de fixer le taux d'intérêt à un niveau qui permet à l'IMF d'assurer son autosuffisance financière et d'être rentable pour pouvoir perdurer indépendamment des subventions et dons. Et la vision welfariste ${ }^{138}$ du bien-être social qui considère que la recherche de la performance financière entraînera intuitivement l'omission de la mission sociale qui est le fondement idéologique du concept micro-crédit.
} 
personnes à bas revenus ont démarré dans les années 1993-1994 ${ }^{139}$. Aussi, la mise en place de programme Micro Start du PNUD ${ }^{140}$ a contribué à l'émergence et au financement de plusieurs institutions de micro-finance.

Le micro-crédit au Maroc est régi par le droit des associations (15 novembre 1958) tel que modifié et complété en 2002, et par la loi 18-97 relative au micro-crédit promulguée par le dahir du 15 février 1999 tel qu'elle été modifiée et complétée par les lois 58-03 du 06 mai 2004 et 41-12 du 28 décembre 2012. De plus, la loi n 34-03 relative aux établissements de crédit et organismes assimilés en ce qui concerne le contrôle de Bank $\mathrm{Al}$ Maghrib $^{141}$ a instaurer les règles de contrôle des associations de micro-crédit.

La loi 58-03 a doté le secteur de deux structures d'encadrement, un Conseil Consultatif pour le Micro-crédit (CCM) qui est consulté sur toutes les questions liées au développement du secteur et la Fédération Nationale des Associations de Micro-crédit (FNAM) qui est un organe de concertation, de coordination interne et de représentation externe.

Les acteurs du micro-crédit au Maroc se répartissent aujourd'hui selon un schéma classique, fait d'IMF, d'institutionnels, de bailleurs de fonds et d'organismes d'appui. De ce fait on compte actuellement $\mathbf{1 3}$ associations ${ }^{142}$ autorisées à exercer une activité de microfinance au Maroc $^{143}$, la Fédération nationale des associations de micro-crédit (FNAM) $^{144}$, le Conseil consultatif du micro-crédit ${ }^{145}$ et le Comité de suivi des activités des associations de micro-crédit (AMC) ${ }^{146}$

139 - Le premier prêt accordé en 1993 par l'AMSED à une femme.

140 - MicroStart est un programme pilote "standa rd" initié par le Programme des Nations Unies pour le Développement (PNUD), qui couvre 30 pays à travers le monde dont 10 en Afrique. Son lancement s'inscrit dans le cadre de la campagne de l'ONU lancée en 1977 visant à donner l'accès aux services de micr-ofinance à 100 millions de foyers les plus pauvres du monde, notamment a ux femmes de ces foyers.

${ }^{141}$ - Le contrôle sur pièces et sur place des associations de micro-crédit, a été transféré à Bank Al-Maghrib au vue de la loi bancaire n 34-03 relative aux établissements de crédit. Les résultats des contrôles effectués par Bank Al-Maghrib sont adressés au Comité de suivi. 142 - BanK Al Maghrib, rapport annuel de l’année 2014.

143 - Association AL Amana pour la Promotion des Microentreprises (ALAMANA), Association Al Karama pour le Micro-Crédit (AL KARAMA), Association Ismailia pour le Micro-Crédit (AIMC), Association Marocaine de Solidarité Sans Frontière (AMSSF), Association Marocaine Oued Serou pour le Micro-Crédit (AMOS), Association Tétouanaise des Initiatives Sociaux- Professionnelles (ATIL), ATTAWFIQ MICRO FINANCE, Fondation «ARDI », Fondation Micro Crédits du Nord, Fondation pour le Développement Local et le Partenariat (FONDEP), TAWADA, BAB RIZK JAMEEL, Institution Marocaine d'Appui à la Micro-Entreprise (INMAA).

144 - Elle est créée entre les adhérents, c'est une entité sous forme d'association à but non lucratif, régie d'une part par la loi sur les associations, telle que modifiée et complétée, et d'autre part par la loi 18-97 relative au Micro crédit.

145 - Présidé par le ministre des finances et regroupe des représentants du Ministère des Finances, de Bank Al Maghrib, de la FNAM, et des banques marocaines. Il a pour mission 
Au Maroc, on compte plus de 810.000 clients avec des encours qui dépassent 4.5 milliards MAD. Les AMC créent plus de 6000 emplois directs permanents déployés dans environ 1300 points de vente $(52.7 \%$ urbain et $47.3 \%$ rural), qui couvrent $60 \%$ des clients en milieu urbain et $40 \%$ en milieu rural. Les AMC ne servent que 10 à $20 \%$ du marché cible qui représente un potentiel client de 3.2 millions.

Avec plus 4.5 milliards de DH d'encours en Mars 2012, les AMC Marocaines dépassent de loin la taille d'une association moyenne. Il est donc normal en quelque sorte que celles-ci désirent accéder plus facilement aux marchés financiers, et prêter des fonds surtout qu'à ce jour, la loi marocaine ne permet que l'octroi de micro-prêts.

La situation actuelle des AMC est assez critique puisque d'une part, il y a ce désir de fusion et de concentration, et d'offre de nouveaux produits et de nouvelles sources de financement. Mais d'autre part, il ne faut pas non plus oublier les avantages que procure le statut juridique d'association à celles-ci : exonérations fiscales, absence d'obligations prudentielles.

\section{La viabilité financière des associations de micro-crédit}

La rentabilité est la capacité d'une entreprise à dégager des bénéfices à partir des moyens mis en œuvre pour y parvenir. La rentabilité financière d'une IMF peut être mesurée grâce à l'analyse de ses performances économiques et financières. Elles sont exprimées par des indicateurs chiffrées qui ont pour objectifs d'apprécier l'efficacité et l'efficience de l'entreprise dans l'utilisation de ses ressources, compte tenu de sa mission et de ses objectifs stratégiques.

Les indicateurs de rentabilité telles les rentabilités sur fonds propres et la rentabilité sur actifs reflètent la performance de l'ensemble des domaines de l'institution.

Pour servir ses clients d'une façon durable, une IMF doit se doter des moyens d'assurer sa propre continuité, c'est-à-dire devenir une organisation pérenne ou viable.

La viabilité financière signifie que les AMC soient rentables financièrement. Pour ce faire elles doivent assurer une couverture des charges par les produits, disposer d'un amortisseur systémique (système d'assurance, caution mutuelle, fonds de garantie,...), d'un système

d'examiner les autorisations d'exercice, le montant maximum des micro-crédits accordés, le taux d'intérêt maximal pratiqué, ainsi que les ratios de solvabilité des IMF.

146 - Composé des représentants de trois départements ministériels : Finances, Intérieur, Emploi et Affaires sociales. Il est chargé de veiller au respect par les associations de microcrédit des dispositions de la loi régissant le micro-crédit et des textes pris pour son application. 
comptable efficace, d’un personnel bien formé, d’un dispositif du contrôle efficace et d'un système d'information et de gestion.

D’après données chiffrées tirées des rapports de BAM de 2007 à 2013, on peut dire que sur 7 années l'encours de crédit est presque stable voir en baisse (4,9 milliards de MAD en 2013 contre 5,5 milliards de MAD en 2007), ce qui constitue un mauvais signe du fait que les AMC octroient moins de crédit à la clientèle. Le taux de créance en souffrance est en moyenne acceptable par rapport au taux des banques environ 5\%. Le résultat net est en moyenne positif (0,2 milliards en 2013) qui montre un signe de rentabilité. Sur la base de ces données, on peut dire que les AMC au Maroc sont généralement rentable financièrement. Mais est ce qu'elles sont socialement performantes?

\section{La performance sociale des IMF : les impacts du micro-crédit}

La performance sociale des institutions de micro-finance est vérifiée à travers l'évaluation d'impact des micro-crédits distribués par les associations de micro-crédit.

L'évaluation de l'impact du micro-crédit sur la pauvreté est le champ d'investigation le plus large qui a attiré beaucoup de chercheurs et qui a fait couler beaucoup d'encre, et ce parce que la principale mission accordée au micro-crédit est la lutte contre la pauvreté.

Toutefois, les études et les publications ayant trait à ce sujet sont parcellaires et n'ont pas une portée générale qui puisse démontrer l'impact du micro-crédit sur la réduction de la pauvreté.

Mais d'autres questions méritent aussi d'être posées à ce sujet. Elles ont fait l'objet de plusieurs études qui s'articulent autour de deux points et pistes de recherches à s'avoir :

- $\quad$ la microfinance atteint-elle les plus pauvres ?

- $\quad$ La microfinance parvient-elle à faire sortir durablement les pauvres de leur pauvreté ?

$\mathrm{Au}$ Maroc pour répondre à ces deux questions nous allons exposer brièvement les résultats de deux études d’impact du micro-crédit sur la population, l'une est conduite la FNAM (organe de concertation) et l'autre par l’Agence Française de développement (AFD) (bailleur de fonds), les résultats seront différent eu égard à la vision de la partie qui a menée l'évaluation d'impact.

Selon l'AFD dans son rapport (2012) ${ }^{147}$ sur l'impact du micro-crédit au milieu rural au Maroc (le cas de l'AMC Al Amana) :

- Le taux d'accès au crédit est limité 16\%,

\footnotetext{
147 - Agence Française de Développement, 2012, « Evaluation de l'impact du microcrédit en zone rurale au Maroc », série d'analyse d'impact.
} 
- Le micro-crédit a un impact sur les activités existantes (agriculture et élevage),

- Le micro-crédit n’a pas d’impact sur la création de nouvelles activités,

- Les effets sur la consommation sont faibles.

- les effets sur le niveau de richesse et de pauvreté, approximé par le niveau de consommation, sont nuls.

Pour l'étude d'impact coordonnée par la FNAM les résultats différent de celle réalisée par l’AFD à savoir que :

- Le micro-crédit permet d'augmenter significativement les profits et les investissements des micro-entreprises .

- Par contre, la création d'une nouvelle activité suite à l'obtention d'un micro-crédit reste mitigée.

- L'augmentation des profits de la micro-entreprise, suite à l'obtention d'un micro-crédit, se retrouve dans l'augmentation des revenus des microentrepreneurs.

- Perceptions fortes de changement :

$\checkmark$ changement positif de l'alimentation au sein du ménage (62\%),

$\checkmark$ d'une autonomie renforcée (68\%)

$\checkmark$ et d'une amélioration du« bien-être » (57\%).

- Perceptions modérées de changement :

$\checkmark$ une proportion significative mais minoritaire de clients témoigne d'un changement positif de l'état de santé (37\%),

$\checkmark$ de l'éducation (36\%)

$\checkmark$ et de la considération du conjoint (45\%) ainsi que des enfants.

Bien qu'elles soient rentables financièrement et performantes socialement, les IMF doivent adopter une stratégie qui leur permet d'assurer une pérennité et un degré de portée sociale élevé. Une IMF doit concilier trois grands objectifs stratégiques à savoir la portée, l'impact et la viabilité. C'est-à-dire que Les IMF doivent être rentable financièrement, performant au niveau social et avoir un impact sur les clients. Ce qui constitue un paradoxe.

\section{Responsabilité sociale versus performance financière : quel arbitrage ?}

C'est légitime pour les IMF de prétendre à une performance financière, tout est mis en œuvre pour afficher de bons résultats financiers. Cependant, une attention trop poussée à la rentabilité risquerait d'éloigner l'institution des pauvres (sa raison d'être) en faisant une sélection trop rigoureuse des clients. Par contre, une vision trop sociale pourrait inciter à l'application des taux d'intérêts trop bas mettant en danger la viabilité de l'institution. En effet, plusieurs IMF, face aux risques élevés que représentent les plus pauvres, ont orienté leur activité vers les plus nantis qui appartiennent aux classes moyennes. 
Autrement dit, on veut savoir si le ciblage des plus pauvres marquerait un frein à l'atteinte de bonnes performances financières ou si plutôt ces deux objectifs peuvent être réalisés conjointement.

Cependant, la meilleure manière d'aider les pauvres à avoir accès aux services financiers oppose l'approche des welfarists ${ }^{148}$ à celle des institutionnalistes $^{149}$. Bien qu'elles partagent l'objectif de réduction de la pauvreté, ces deux approches placent la microfinance à la croisée des chemins.

L'approche respective des welfarists et des institutionnalistes a fait l'objet d'un certain nombre de critiques. La première approche est confrontée au problème de viabilité et de pérennité induit par les subventions, la faiblesse des taux de remboursement et l'augmentation des coûts de fonctionnement ; la deuxième approche a pour clientèle de prédilection les micro-entrepreneurs très proches de la ligne de pauvreté auxquels sont appliqués des taux d’intérêt assez élevés pour assurer l'autonomie financière des IMF. La microfinance doit s'assurer de respecter ses propres " fondamentaux " pour limiter les dérives d'une approche purement commerciale.

Le paradigme de la pérennité financière fait désormais consensus au niveau de la plupart des acteurs du secteur : selon ce paradigme, la microfinance aurait vocation, pour assurer sa viabilité économique et massifier sa couverture, à recouvrer ses coûts mais aussi à produire des bénéfices pour assumer son extension et attirer des capitaux privés. Cette évolution peut les conduire à se tourner vers des segments de clientèle plus

\footnotetext{
148 - Les welfarists se fondent sur la théorie de responsabilité sociale vis-à-vis de la clientèle afin de de répondre à ses attentes. Cette école de pensée évalue la performance de l’IMF du point de vue du client à travers la portée sociale et l'analyse d'impact : elle cible les plus pauvres dont les revenus sont à 50\% inférieurs au seuil de pauvreté (2\$ par jour) et vise à améliorer leurs conditions de vie. Elle est composée essentiellement d'institutions solidaires - ONG ou coopératives - qui considèrent la micro finance comme un moyen clé pour réduire la pauvreté des plus pauvres.

149 - Les institutionnalistes se fondent sur la théorie des contrats qui considère que l'incomplétude des contrats peut conduire à des comportements opportunistes des demandeurs de crédits. Les institutionnalistes évaluent la performance du point de vue de l'institution en ciblant une clientèle de ménages pauvres et en visant la pérennité financière de l'IMF. Ils ont conçu un ensemble de "meilleures pratiques" bancaires afin d'accroître l'efficacité des systèmes de gestion, dont l'adoption est une étape essentielle pour atteindre l'autosuffisance financière à l'échelle industrielle et avoir accès au marché financier. Ils considèrent l'autonomie financière comme un critère qui remplit au mieux la mission sociale. Ils représentent essentiellement des institutions financières : soit des institutions spécialisées en micro finance réglementées qui s’inscrivent clairement dans une logique de rentabilité, soit des caisses villageoises ou certaines banques commerciales traditionnelles qui se sont plus récemment Impliquées dans la microfinance.
} 
rentables, c'est-à-dire essentiellement urbains et moins pauvres, sans autres alternatives pour les ménages les plus exclus.

\section{Quelle stratégie de l’équilibre pour les IMF ?}

On a mentionné deux objectifs auxquels sont confrontées les IMF (performance sociale et rentabilité financière), il existe néanmoins un troisième qui est l'impact que peuvent avoir les IMF. La meilleure stratégie que puisse avoir une institution de micro-finance est de concilier ses trois grands objectifs stratégique à savoir la portée ( atteindre un nombre important de clients), la viabilité financière ( construire une organisation viable pour être apte à continuer dans la durée) et pour finir l'impact (apporter à chaque client un service correspondant à ses besoins et lui donnant satisfaction .

Il peut exister une synergie entre ces trois objectifs propres à la micro-finance. Par exemple, si l'impact sur les clients est bon, de plus en plus de personnes seront désireuses de devenir clientes d'où une augmentation de la portée. Un impact satisfaisant sur les clients montre que leur demande de crédit a été porteuse et génératrice de revenus, par conséquent il y aura de fortes volontés et capacités de rembourser les crédits. Cet impact est d'autant plus amplifié dans la mesure où les clients potentiels rassurés et confiants peuvent épargner davantage en toute sécurité.

Cette croissance permettra des économies d’échelle, renforçant la viabilité financière. La bonne santé financière séduit les bailleurs de fonds et permet d'acquérir davantage de financement, ce qui renforcera encore plus la croissance. Plus l'IMF montrera de bons signes de pérennité, plus les clients voudront $\mathrm{y}$ adhérer. De bons résultats financiers permettre d'avoir suffisamment de fonds pour lancer par exemple de nouveaux produits, et donc renforcer l'impact.

La recherche de la viabilité financière pousse les IMF à prêter plus d'attention aux exigences de leurs clients, ce qui va générer des produits financiers supplémentaire et de meilleurs bénéfices économiques pour les clients et par conséquent un meilleur impact.

Bien que les trois objectifs stratégiques puissent être complémentaires, dans certains cas ils entrent en conflits et de risques c'est à ce moment qu'apparaissent des arbitrages auxquels sont confrontées les IMF.

Il se pourrait que la priorité donnée à la portée et non à la croissance quantitative puisse se faire au détriment de la qualité des services rendus et non affecter l'impact, si les efforts sont mis également sur la croissance et qu'ils ne s'accompagnent pas d'une bonne structuration de l'institution, cela peut créer un effet inverse : une crise de croissance qui s’accompagne d'une 
perte de contrôle de l'organisation et une dégradation des taux de remboursement.

Enfin, si la priorité est donnée à l'atteinte de la viabilité financière, on risque de privilégier les prêts des montants de plus en plus élevés à des clients de moins en moins pauvres, il y a un risque de dérive par rapport à la mission originale de l'IMF.

Il peut exister aussi un compromis à faire entre l'impact et la portée. En fait les IMF peuvent choisir de mettre l'accent sur le nombre des personnes qu'elles veulent toucher plutôt que le type de personnes. Ainsi, ces banques des pauvres adapteront leurs services à une clientèle faisant partie de la catégorie des moins pauvres voire dans certains cas une clientèle constituée de propriétaire de PME. Par conséquent, elles délaissent une fois de plus les plus nécessiteux. Ce choix pourrait s'expliquer par le fait qu'il existe encore des doutes sur le pouvoir de la microfinance de sortir les plus pauvres des pauvres de leur extrême précarité.

\section{Conclusion}

Le secteur du microcrédit au Maroc est considéré comme un leader dans le Moyen-Orient et dans l'Afrique du Nord. Ce succès a été largement attribué à l'appui des pouvoirs publics marocains qui ont mis à la disposition du secteur un cadre réglementaire favorable, ainsi que par l'attrait de bailleurs de fonds étrangers qui ont soutenu la croissance du secteur. Cette croissance a été alimentée par une forte demande pour les services financiers des populations exclues du système bancaire et par l'existence d'un potentiel d'activités génératrices de revenus au Maroc avec des gros besoins de financement et d'assistance technique.

Les institutions de microfinances au Maroc ont été toujours classées parmi les plus performantes dans le monde grâce à la croissance du secteur qui a fait du Maroc une référence dans le domaine du microcrédit.

Pour éviter toute dérive, il est important de reconsidérer le cadre général et les dispositifs de contrôle interne comme une composante d'une politique globale de gestion et de prévention des risques. Il faut intégrer les visites des clients dans les dispositifs de contrôle pour vérifier leur situation d'endettement, l'utilisation du prêt par ces clients et la situation de leur activité et enfin identifier les risques des erreurs de gestion et de fraudes.

En tant qu'acteur d'inclusion financière et de lutte contre la pauvreté, les pouvoirs publics doivent instaurer et standardiser les méthodes d'évaluation d'impact de l'intervention des IMF sur les bénéficiaires pour légitimer l'action. Certaines Institutions affichent comme mission la lutte contre l'exclusion financière, d'autres la lutte contre la pauvreté. Cependant la pauvreté est une problématique très complexe que la microfinance à elle seule ne peut prétendre résoudre ou remplacer l'action de l'Etat et celle des 
politiques publiques, et par conséquent il ne faut pas accuser la microfinance si elle n'arrive pas à résorber les problèmes de pauvreté.

A noter aussi que certains considèrent les associations de microcrédit comme de nouveaux usuriers qui exploitent l'incapacité des plus pauvres à résister à la tentation de l'emprunt, ils soulignent que les taux d'intérêt, souvent présentés de manière obscure, restent très élevés par rapport à ce que paient les plus riches. Ces critiques attribuent même au microcrédit les suicides de paysans surendettés, soumis à une pression indécente exercée par les agents de prêts. En effet, ceux-ci, rémunérés en fonction du nombre de clients et de leur taux de remboursement, sont incités à pousser à l'emprunt d'abord, et à forcer au remboursement par tous les moyens.

A signaler enfin que la rentabilité financière contre la performance sociale est un faux débat. Il faut que la conception et le pilotage d'une IMF doit être basé sur une approche entrepreneuriale, on doit veiller à bien travailler le passage d'un projet à l'entreprise par la création d'entreprise et il faut que l'action du gouvernement prévoie des mesures incitatives pour les institutions opérant dans les zones défavorables.

\section{References:}

Agence canadienne de développement international, 1999, "Guide de référence pour le secteur de la microfinance », 126 p.

Agence Française de Développement, 2012, «Evaluation de l'impact du microcrédit en zone rurale au Maroc », série d'analyse d'impact, 51 p.

Amouroux Philippe, "La finance solidaire pour un autre projet de société. », Revue du MAUSS 1/2003 (n $\left.{ }^{0} 21\right)$, p. 66-72.

Association de micro-crédit AL AMANA, 2008, «Etude d'impact des programmes de micro-crédit au Maroc ».

Bank Al Maghrib, Rapports de la supervision bancaire, 2007 à 2013.

Bank Al Maghrib, statistiques monétaires, 2007 à 2013.

BanK Al Maghrib, rapports annuels des années 2007 à 2014.

Bedecarrats Florent, "Évaluer la microfinance, entre utilité sociale et performances financières. », Revue Française de Socio-Économie 2/2010 (nº 6), p. 87-107.

Bedecarrats Florent, 2012, «La microfinance entre l'utilité sociale et performances financières ", thèse de doctorat, université de Paris panthéonSorbonne, $418 \mathrm{p}$.

Boyé Sébastien, Hajdenberg Jérémy, Poursat Christine «Le guide de la microfinance : Microcrédit et épargne pour le développement », Éditions d'Organisation, 2009.

CREUSOT Anne-Claude, POURSAT Christine, «Pour une meilleure inclusion financière : renforcer les institutions intermédiaires», Revue Tiers Monde 1/2009 (n 197), p. 17-36. 
Fédération Nationale des Associations de Micro-crédit (FNAM), « Evaluation de l'impact de la microfinance au Maroc ».

Guérin Isabelle, Lapenu Cécile, Doligez François, «La micro-finance estelle socialement responsable ?», Revue Tiers Monde 1/2009 (n 197), p. 516.

Laurent Lhériau, 2009, «Précis de réglementation de la microfinance », Agence Française de Développement.

Loi n³4-03 relative aux établissements de crédit et organismes assimilés Promulguée par le dahir n 1-05-178 du 15 Moharrem 1427 (14 février 2006).

LAPENU Cécile, et autres, «Performances sociales: Une raison d'être des institutions de microfinance et pourtant encore peu mesurées. Quelques pistes. », Mondes en développement 2/2004 ( $\mathrm{n}^{0}$ 126), p. 51-68.

LAPENU Cécil., 2007, « Performance sociale versus performance financière : peut-on être rentable en s'adressant à des populations pauvres ? », Horizons Bancaires n 334, décembre 2007, pp. 45-54.

Mayoux L. (1998), «L’empowerment des femmes contre la viabilité ? versus un nouveau paradigme dans les programmes de micro-crédit », in Les silences pudiques de l'économie, Économie et rapports sociaux entre hommes et femmes, pp.73-97.

Servet Jean-Michel, « Responsabilité sociale versus performances sociales en microfinance», Revue Tiers Monde 1/2009 (n 197), p. 55-70. 